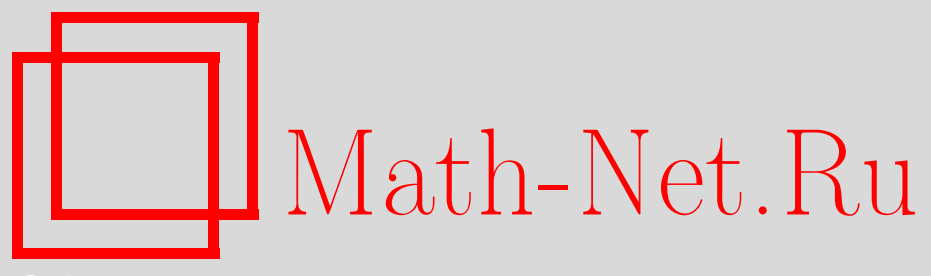

А. Э. Хузиева, Д. А. Шабанов, Количественные оценки характеристик в гиперграфах с большим обхватом и большим хроматическим числом, Матем. заметки, 2015, том 98, выпуск 6, 948-951

DOI: https://doi.org/10.4213/mzm10979

Использование Общероссийского математического портала Math-Net.Ru подразумевает, что вы прочитали и согласны с пользовательским соглашением http://www . mathnet.ru/rus/agreement

Параметры загрузки:

IP: 3.80 .253 .173

26 апреля 2023 г., 17:31:15

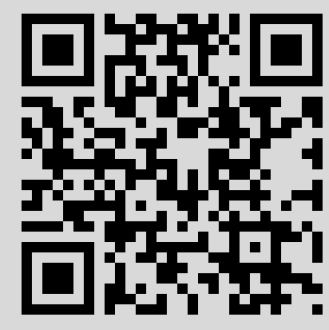




\section{Количественные оценки характеристик в гиперграфах с большим обхватом и большим хроматическим числом}

\section{А. Э. Хузиева, Д. А. Шабанов}

1. История задачи. Работа посвящена изучению известной проблемы экстремальной комбинаторики, связанной с раскрасками гиперграфов. Напомним основные определения из теории гиперграфов.

Гиперграфом $H$ называется пара множеств $H=(V, E)$, где $V=V(H)$ - это некоторое конечное множество, элементы которого называются вершинами гиперграфа, а $E=E(H)$ произвольная совокупность подмножеств $V$, которые принято называть ребрами гипергра$\oint a H$. Если каждое ребро $A \in E$ состоит ровно из $n$ вершин, то говорят, что гиперграф $H$ является $n$-однородным. Раскраской множества вершин гиперграфа $H=(V, E)$ называется любое отображение $f: V \rightarrow \mathbb{N}$. Раскраска $f$ является правильной для гиперграфа, если в ней нет одноцветных ребер. Минимальное число цветов, необходимое для правильной раскраски гиперграфа $H$, называется его хроматическим числом и обозначается $\chi(H)$.

Cтепенъю вершины $v$ гиперграфа $H$ называется количество ребер $H$, содержащих $v$. Максимальную степень вершины гиперграфа $H$ мы будем обозначать через $\Delta(H)$. Циклом длины $k$ в гиперграфе $H=(V, E)$ называется чередующаяся последовательность $v_{0}, A_{1}, v_{1}$, $\ldots, A_{k}, v_{k}=v_{0}$ из $k$ различных вершин $v_{0}, \ldots, v_{k-1}$ и $k$ различных ребер $A_{1}, \ldots, A_{k}$ с условием, что для любого $i=1, \ldots, k$ выполнено $v_{i-1} \in A_{i}$ и $v_{i} \in A_{i}$. Длина минимального цикла в гиперграфе называется его обхватом. Мы будем обозначать обхват гиперграфа $H$ через $g(H)$. Гиперграфы с обхватом больше двух принято называть простыми.

В 1973 г. Эрдеш и Ловас в своей работе [1] доказали, что для любых $n \geqslant 3, r \geqslant 2$, $s \geqslant 2$ существует $n$-однородный гиперграф $H$ с хроматическим числом больше $r$ и обхватом больше $s$. Более того, они показали, что можно разумно ограничить число ребер и максимальную степень вершины в таком гиперграфе.

Теорема 1 (Эрдеш, Ловас, [1]). Пусть $n \geqslant 3, r \geqslant 2, s \geqslant 2$. Тогда существует $n$-однородный гиперграф $Н$ со следующими свойствами:

$$
\chi(H)>r, \quad g(H)>s, \quad|E(H)| \leqslant 4 \cdot 20^{s} n^{3 s-2} r^{(n+1) s}, \quad \Delta(H) \leqslant 20 n^{2} r^{n+1} .
$$

Тем самым, Эрдеш и Ловас мотивировали изучение экстремальной величины $m(n, r, s)$, определяемой как минималъно возможное количество ребер гиперграфа в классе $n$-однородных гиперграфов с хроматическим числом больше $r$ и обхватом больше $s$. Из теоремы 1 следует, что

$$
m(n, r, s) \leqslant 4 \cdot 20^{s} n^{3 s-2} r^{(n+1) s} .
$$

Асимптотическое поведение величины $m(n, r, s)$ достаточно хорошо изучено в случаях $s=1$ (условие на обхват тривиально) и $s=2$ (класс простых гиперграфов). Подробнее об этом можно прочитать, например, в обзоре [2]. Для гиперграфов с более сильными ограничениями на обхват задача изучена намного хуже. Верхнюю оценку для $m(n, r, s)$ Эрдеша-Ловаса из теоремы 1 для произвольного $s$ можно уточнить с помощью конструкции гиперграфов с большим обхватом и большим хроматическим числом Косточки и Рёдля из [3]. Их идеи в сочетании с теоремой Сауера [4] дают более сильную верхнюю оценку нежели теорема 1 при фиксированных $n, s \geqslant 3$ и растущем $r$ :

$$
m(n, r, s) \leqslant 2 \cdot 4^{s+2} n^{3 s+3} r^{s n+2}(\ln r)^{s} .
$$

Работа выполнена при поддержке Программы поддержки ведущих научных школ (грант № НШ-2964.2014.1) и гранта Президента РФ МК-692.2014.1.

DOI: $10.4213 / \mathrm{mzm} 10979$ 
Единственная же нетривиальная нижняя оценка $m(n, r, s)$ для $s>2$ была получена Косточкой и Кумбхатом в работе [5]. Они показали, что для любых $s, r \geqslant 2, \varepsilon>0$ найдется такое $n_{0}=n_{0}(r, s, \varepsilon)$, что при всех $n>n_{0}$

$$
\begin{array}{ll}
m(n, r, s)>r^{n(\lfloor s / 2\rfloor+1)} n^{1-\varepsilon} & \text { при нечетном } s ; \\
m(n, r, s)>r^{n(\lfloor s / 2\rfloor+1)} n^{-\varepsilon} & \text { при четном } s .
\end{array}
$$

Заметим, что зазор между оценками (1) и (2) имеет экспоненциальный порядок роста по $n$ при фиксированных $r \geqslant 2, s>2$. Тем самым, неизвестна даже асимптотика логарифма величины $m(n, r, s)$.

2. Новые результаты. В настоящей работе мы улучшаем нижние оценки 2 для $m(n, r, s)$. Новые оценки получены с помощью некоторого структурного результата о количественных характеристиках гиперграфов с большим обхватом и большим хроматическим числом. Для его точной формулировки введем следующие определения. Пусть $H=(V, E)-$ $n$-однородный гиперграф, а $\delta$ - некоторое положительное число. Вершина $v \in V$ называется $\delta$-легкой, если ее степень в гиперграфе $H$ не превосходит $\delta$. В противном случае вершина называется $\delta$-тлжелой. Ребро называется $\delta$-тяжельм, если в нем более половины вершин являются $\delta$-тяжелыми. В противном случае ребро называется $\delta$-легким. Тогда выполнена следующая теорема.

Теорема 2. Пусть $\alpha>0$ - фиксированное число. Тогда существуют такие положительные $c=c(\alpha)$ и $n_{0}=n_{0}(\alpha)$, что при $r \geqslant 2, \delta=c \cdot r^{n-1}$ u $n>n_{0}$ для любого $n$-однородного гиперграфа $H=(V, E)$ с обхватом больше пяти и условиями

1) $\Delta(H) \leqslant n^{\alpha} r^{n-1}$;

2) каждая вершина $H$ содержится не более чем в $c \cdot r^{n-1} \delta$-тяжелых ребрах; выполнено $\chi(H) \leqslant r$.

Смысл теоремы 2 состоит в том, что если гиперграф $H$ не является $r$-раскрашиваемым, то либо в нем найдется вершина уж очень большой степени, не менее $n^{\alpha} r^{n-1}$, либо найдется вершина, в окрестности которой найдется много, порядка $c r^{n-1}$, вершин просто большой степени, не меньше $c r^{n-1}$.

Количественные оценки в теореме 2 достаточно близки к максимально возможным. Косточка и Рёдль показали (см. [3]), что существуют $n$-однородные гиперграфы с хроматическим числом больше $r$, со сколь угодно большим обхватом и максимальной степенью вершины не более $\left\lceil n r^{n-1} \ln r\right\rceil$. Тем самым, в формулировке теоремы величина $\delta$ не может превышать $n r^{n-1} \ln r$, т.е. не более чем в $n \ln r$ раз больше, чем в нашей теореме.

В качестве следствия мы получаем улучшение нижних оценок $(2)$ величины $m(n, r, s)$.

Следствие 1. Существует такая абсолютная константа с >0, что для $5 \leqslant s<n / 2$ выполнено

$$
\begin{array}{ll}
m(n, r, s) \geqslant c^{\lfloor s / 2\rfloor} r^{(n-s)(\lfloor s / 2\rfloor+1)} & \text { при четном } s ; \\
m(n, r, s) \geqslant c^{\lfloor s / 2\rfloor} n r^{(n-s)(\lfloor s / 2\rfloor+1)} & \text { при нечетном } s .
\end{array}
$$

3. Идея доказательства. В качестве основного метода доказательства нами используется метод случайной перекраски. В нем мы следуем

- идеям Косточки и Кумбхата из [5] относительно двухэтапной перекраски;

- построению раскраски из работы Купавского и Шабанова [6];

- технике вероятностного анализа из работы Козика и Шабанова [7].

Итак, пусть $n$-однородный гиперграф $H=(V, E)$ удовлетворяет условиям теоремы 2. Нам необходимо доказать существование правильной раскраски множества вершин гиперграфа $H$ в $r$ цветов. Для этого мы построим некоторую случайную $r$-раскраску $V$ и покажем, 
что она является правильной с положительной вероятностью. Без ограничения общности будем считать, что $V=\{1, \ldots, N\}$. Также всюду далее $\delta$-тяжелые ( $\delta$-легкие) вершины и ребра будем называть просто тяжелыми и легкими. Наконец, для каждого ребра $A$ через $A_{R}$ мы будем обозначать множество его тяжелых вершин, а через $A_{L}-$ множество его легких вершин.

Опишем конструкцию с помощью следующего рандомизированного алгоритма.

Стартуем с равномерной случайной раскраски множества вершин в $r$ цветов: вершине $v$ присваиваем цвет $\xi_{v}$, где $\left\{\xi_{1}, \ldots, \xi_{N}\right\}$ - независимые случайные величины с равномерным распределением на множестве цветов $\{1, \ldots, r\}$. Также введем независимые случайные величины, которые нам понадобятся далее: $\left\{\eta_{1}, \ldots, \eta_{N}\right\}$ со следующим условным распределением на множестве цветов $\{1, \ldots, r\}$ :

$$
\mathrm{P}\left(\eta_{v}=\alpha \mid \xi_{v}=\beta\right)=\frac{1}{r-1} \quad \text { для любых } \quad \alpha \neq \beta \in\{1, \ldots, r\} .
$$

В случайной раскраске $\xi=\left(\xi_{1}, \ldots, \xi_{N}\right)$ гиперграф $H$ может содержать одноцветные и почти одноцветные ребра. Ребро $A$ называется почти одноцветным с доминирующим иветом $\alpha$ в раскраске $\xi$, если

$$
n-s \leqslant \sum_{v \in A} I\left\{\xi_{v}=\alpha\right\} \leqslant n-1,
$$

где $1 \leqslant s<n / 2-$ первый параметр нашей конструкции.

Будем осуществлять следующий алгоритм случайной перекраски, в рамках которого мы хотели бы исправить одноцветные ребра, но так, чтобы почти одноцветные ребра не стали одноцветными доминирующего цвета. Зададим случайный порядок вершин $\sigma$ с помощью вариационного ряда независимых случайных величин $\left\{X_{v}, v \in V\right\}$ (независимых также с набором $\left.\left(\xi_{v}, \eta_{v}, v \in V\right)\right)$ с равномерным распределением на отрезке $[0,1]$. Для ребра $A$ и вершины $v$ будем использовать обозначение

$$
\sigma(v, A)=\sum_{w \in A} I\left\{X_{w} \leqslant X_{v}\right\}
$$

для номера вершины $v$ в ребре $A$.

На первом этапе процесса случайной перекраски будем перекрашивать только тяжелые вершины гиперграфа $H$ и исправлять только тяжелые одноцветные ребра. Будем рассматривать тяжелые вершины в порядке, заданном $\sigma$, и для текущей вершины $v$ проверять следующие условия перекраски.

Условие $1 \mathrm{~h}$. Существует такое тяжелое ребро $A, v \in A$, что $A$ - одноцветно в $\xi$, на момент рассмотрения $v$ ни одна вершина $A$ еще не сменила цвет и, кроме того,

$$
\sigma\left(A_{R}, v\right) \leqslant h
$$

(здесь $1 \leqslant h<n / 2$ - второй параметр конструкции).

Условие $2 \mathrm{~h}$. Не существует такого тяжелого ребра $B, v \in B$, почти одноцветного в $\xi$, что на момент рассмотрения $v$ эта вершина осталась единственной, не покрашенной в $\alpha-$ доминирующий цвет ребра $B$, и $\eta_{v}=\alpha$.

Если условия перекраски выполнены, то присваиваем вершине $v$ цвет $\eta_{v}$. В противном случае, не перекрашиваем $v$ и переходим к следующей вершине.

Получившуюся в результате перекраски тяжелых вершин случайную раскраску обозначим через $\zeta=\left(\zeta_{1}, \ldots, \zeta_{N}\right)$. Во время второго этапа перекраски мы будем перекрашивать только легкие вершины.

Будем рассматривать легкие вершины в порядке, заданном $\sigma$, и для текущей вершины $w$ проверять следующие условия перекраски: 
Условие 11. Существует такое легкое ребро $A, w \in A$, что $A$ - одноцветно или почти одноцветно в $\xi$, одноцветно в $\zeta$, на момент рассмотрения $w$ ни одна легкая вершина $A$ еще не сменила цвет и, кроме того,

$$
\sigma\left(A_{L}, v\right) \leqslant h .
$$

УСловиЕ 2l. Не существует такого ребра $B, w \in B$, почти одноцветного в $\xi$, что на момент рассмотрения $w$ эта вершина осталась единственной, не покрашенной в $\alpha$ - доминирующий цвет ребра $B$, и $\eta_{w}=\alpha$.

Если условие перекраски выполнено, то присваиваем вершине $v$ цвет $\eta_{v}$. В противном случае, не перекрашиваем $v$ и переходим к следующей вершине.

Выбирая параметры конструкции $h=\lceil\ln n\rceil$ и $s=\left\lceil n^{1 / 2}\right\rceil$ и применяя Локальную лемму, мы показываем, что с положительной вероятностью:

1) после первого этапа перекраски все одноцветные в $\xi$ тяжелые ребра перестанут быть одноцветными того же цвета;

2) после второго этапа перекраски все одноцветные в $\zeta$ легкие ребра, которые были одноцветными того же цвета или почти одноцветными с тем же доминирующим цветом в начальной раскраске $\xi$, будут исправлены;

3) в итоговой раскраске не появится одноцветных ребер, которые в $\xi$ не были одноцветными того же цвета или почти одноцветными с тем же доминирующим цветом.

\section{СПИСОК ЦИТИРОВАННОЙ ЛИТЕРАТУРЫ}

[1] P. Erdős, L. Lovász, Infinite and Finite Sets, Vol. II, Colloq. Math. Soc. Janos Bolyai, 10, North Holland, Amsterdam, 1975, 609-627. [2] А. М. Райгородский, Д. А. Шабанов, УМН, 66:5 (2011), 109-182. [3] A. V. Kostochka, V. Rödl, Random Structures Algorithms, 36:1 (2010), 46-56. [4] N. Sauer, J. Combin. Theory, 9:2 (1970), 144-147. [5] A. V. Kostochka, M. Kubmhat, Random Structures Algorithms, 35:3 (2009), 348-368. [6] А. Б. Купавский, Д. А. Шабанов, Докл. РАН, 443:4 (2012), 422-426. [7] J. Kozik, D. Shabanov, J. Combin. Theory. Ser. B (to appear).

\section{А.Э. Хузиева}

Поступило

Московский физико-технический

институт (государственный университет)

E-mail: atomochek@gmail.com

\section{Д. А. Шабанов}

Московский государственный университет имени М.В.Ломоносова;

Московский физико-технический институт (государственный университет)

E-mail: dm.shabanov.msu@gmail.com 